\title{
Diagnosis of a T-lineage acute lymphoblastic leukemia through digitalized cell analysis of the pleural effusion
}

This article was published in the following Dove Press journal:

International Medical Case Reports Journal

31 October 2013

Number of times this article has been viewed

\author{
Benedetta Peruzzi' \\ Ilaria Cutini² \\ Anna Maria Grazia Gelli' \\ Tommaso Rondelli' \\ Marinella Statello' \\ Sara Bencini \\ Francesco Mannelli ${ }^{2}$ \\ Roberto Caporale' \\ Alberto Bosi² \\ Alessandra Fanelli' \\ 'General Laboratory Unit \\ (Microscopy and Clinical Cytometry \\ Unit), ${ }^{2}$ Hematology Unit, Azienda \\ Ospedaliero-Universitaria Careggi, \\ Firenze, Italy
}

Introduction: Pleural effusion as the first clinical manifestation of acute lymphoblastic leukemia (ALL) is a relatively rare event. An early and accurate diagnosis of this clinical picture is very important for adequate patient management.

Case presentation: We report the atypical onset of T-lineage ALL in a 31-year-old man. The patient was admitted to the emergency room due to lung failure; at that moment, the patient's initial blood count was normal; the chest X-ray radiography showed a massive pleural effusion and a thoracentesis was carried out. Routine investigations performed on the pleural fluid using a new technology system and digitalized cell analysis demonstrated infiltration by immature cells. Therefore, bone marrow aspirate and flow cytometry analyses were performed, leading to the diagnosis of T-lineage ALL. A cord blood transplantation procedure was performed at the first hematological remission following chemotherapy regimens. The patient died of septic shock.

Conclusion: The case we reported underlines the usefulness of using automated instruments to identify abnormal lymphoid cells in body fluids.

Keywords: pleural effusion, digital morphology, leukemia

\section{Introduction}

The clinical presentation of some hematological diseases can be challenging, especially in the absence of the characteristic clinical signs of the disease. In the majority of patients admitted to the emergency room with lung failure, it is possible to find congestive heart failure, pneumonia, cancer, pulmonary embolus, tuberculosis, collagenoses, and other such conditions. Most of these signs are associated with pleural effusion, which is often sampled in order to get a diagnosis. Good interaction between laboratory and clinical advice urgency-emergency can be decisive in the early diagnosis of some diseases. The analysis of body fluids for cell counts and differential white blood cells information remains a challenging and time-consuming manual process in many laboratories.

\section{Case presentation}

A 31-year-old man was admitted to the emergency room in August 2012 due to chest pain, dyspnea, cough, and fever. The patient did not have a medical history of night sweats, weight loss, or bleeding. The patient's initial blood count was normal (white blood cell count: $8.55 \times 10^{9} / \mathrm{L}$; red blood cell count: $4.71 \times 10^{12} / \mathrm{L}$; hemoglobin: $14.3 \mathrm{~g} / \mathrm{dL}$; and platelet count: $194 \times 10^{12} / \mathrm{L}$ ). No lymph node enlargement was detectable upon physical examination. The electrocardiogram was normal; the echocardiogram showed pericardial effusion and a chest X-ray revealed bilateral pleural effusion
Largo Brambilla

General Laboratory Unit (Microscopy

and Clinical Cytometry Unit), Azienda

Ospedaliero-Universitaria Careggi,

350134 Firenze, Italy

Tel +39055 7949023

Fax +390557949676

Email peruzzib@aou-careggi.toscana.it 


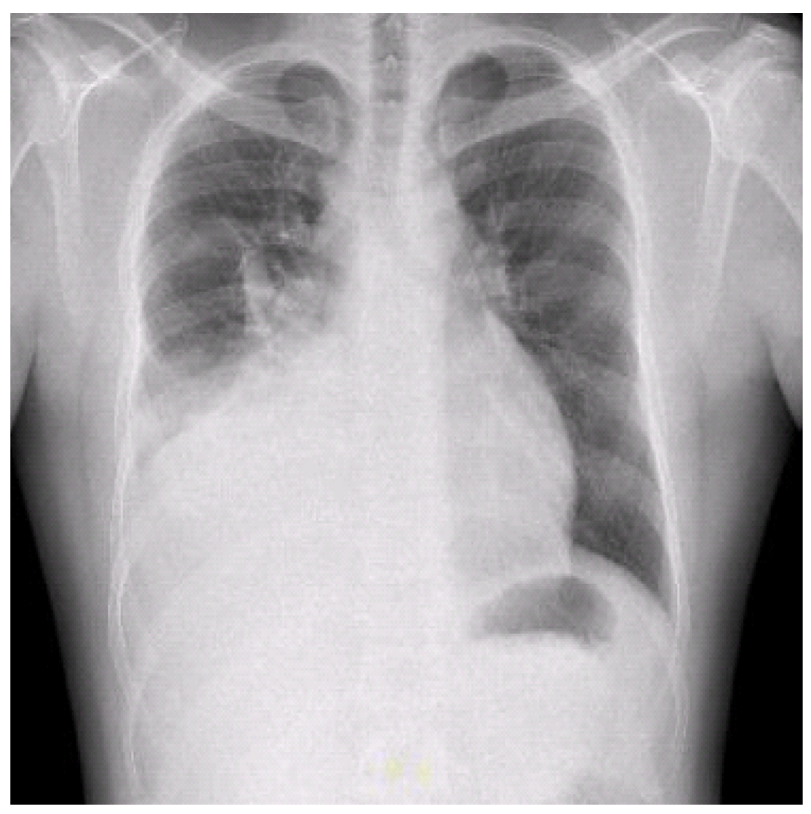

Figure I Chest X-ray revealed a pleural effusion.

(Figure 1). Due to the fact that the patient's general status was worsening, a thoracentesis was required. The fluid from the pleural effusion was sent to the laboratory for routine investigation.

The Sysmex XE-5000 ${ }^{\mathrm{TM}}$ (Sysmex Corporation, Kobe, Japan) hematology analyzer, equipped with the body fluid module, was used as previously described ${ }^{1}$ to obtain the patient's cell count, and to achieve white blood cell differentiation into mononuclear (MN) and polymorphonuclear cells; we obtained a cell count of 14,000 cells $/ \mu \mathrm{L}(90 \%$ MN cells, and $10 \%$ polymorphonuclear cells). Cell cluster spreading from the $\mathrm{MN}$ area into the high-fluorescence area was observed (Figure 2A); this cell cluster had higher forward and side scatter than normal cells (Figure 2B), meaning that those cells were bigger and more complex. Cytospin preparation (CytoFuge2; IRIS International, Chatsworth, CA, USA) was performed with the pleural fluid using $250-300 \mu \mathrm{L}$ of the cell suspension $(20$ cells $/ \mu \mathrm{L})$ to obtain a cell pellet of approximately 5,000 cells. The slides were air-dried and stained with the modified Wright-Giemsa method. Slides were analyzed using the CellaVision ${ }^{\circledR}$ DM96 (CellaVision AB, Lund, Sweden). ${ }^{2}$ Morphological examination (Figure 3) of the pleural fluid revealed a massive infiltration of immature cells, with large and clefted nuclei; the cytoplasm was basophilic without granules, and was scant in volume, large in size, presenting with fine chromatin and evident nucleoli. To clarify the nature of the immature cells observed in the pleural fluid, flow cytometry analysis was performed. A panel of antibodies including surface CD3 (CD3), cytoplasmic CD3 (CD3Cy), CD7, CD4, CD8, CD2, CD5, cytoplasmic TdT (TdTCy), CD45, and CD1a was used. The analysis was performed using a FACSCanto ${ }^{\mathrm{TM}}$ II flow cytometer (BD Biosciences, San Jose, CA, USA). Cytograms (Figure 4) showed that the cells (in red; P1) were CD45+/-, CD3-, CD7++, CD5+, CD2-, CD34+/-, CD1a-, CD3Cy+, $\mathrm{TdTCy}+$, which were immature T-lineage cells. Therefore, a bone marrow aspirate was performed (Figure 5), which showed an infiltration of lymphoblast cells. Flow cytometry analysis on peripheral blood and bone marrow (Figure 6) were also performed; according to the European Group for the Immunological Classification of Leukemia (EGIL) cri-
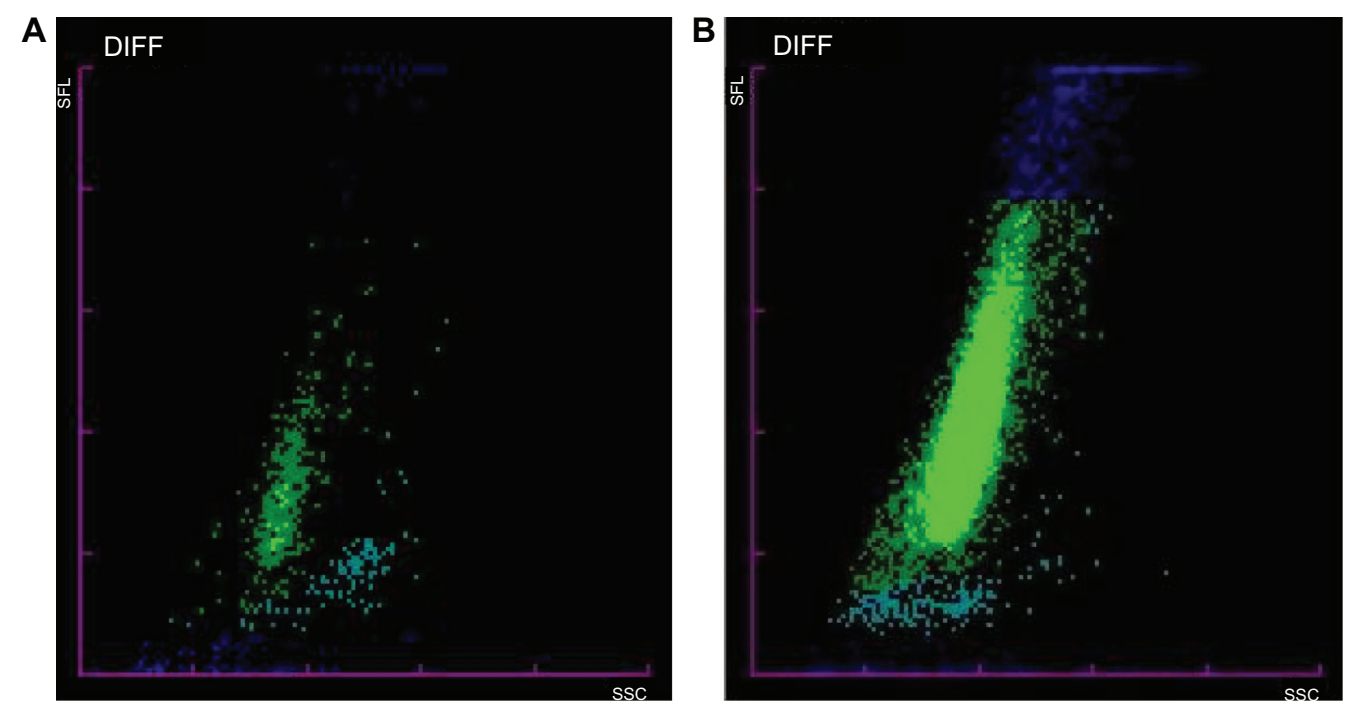

Figure 2 Scattergram of normal and T-ALL pleural fluid.

Note: Scattergram of (A) normal and (B) T-ALL pleural fluid.

Abbreviations: T-ALL, T-lineage acute lymphoblastic leukemia; DIFF, differentiated by flow cytometry; SFL, side fluorescence; SSC, side scatter cells. 

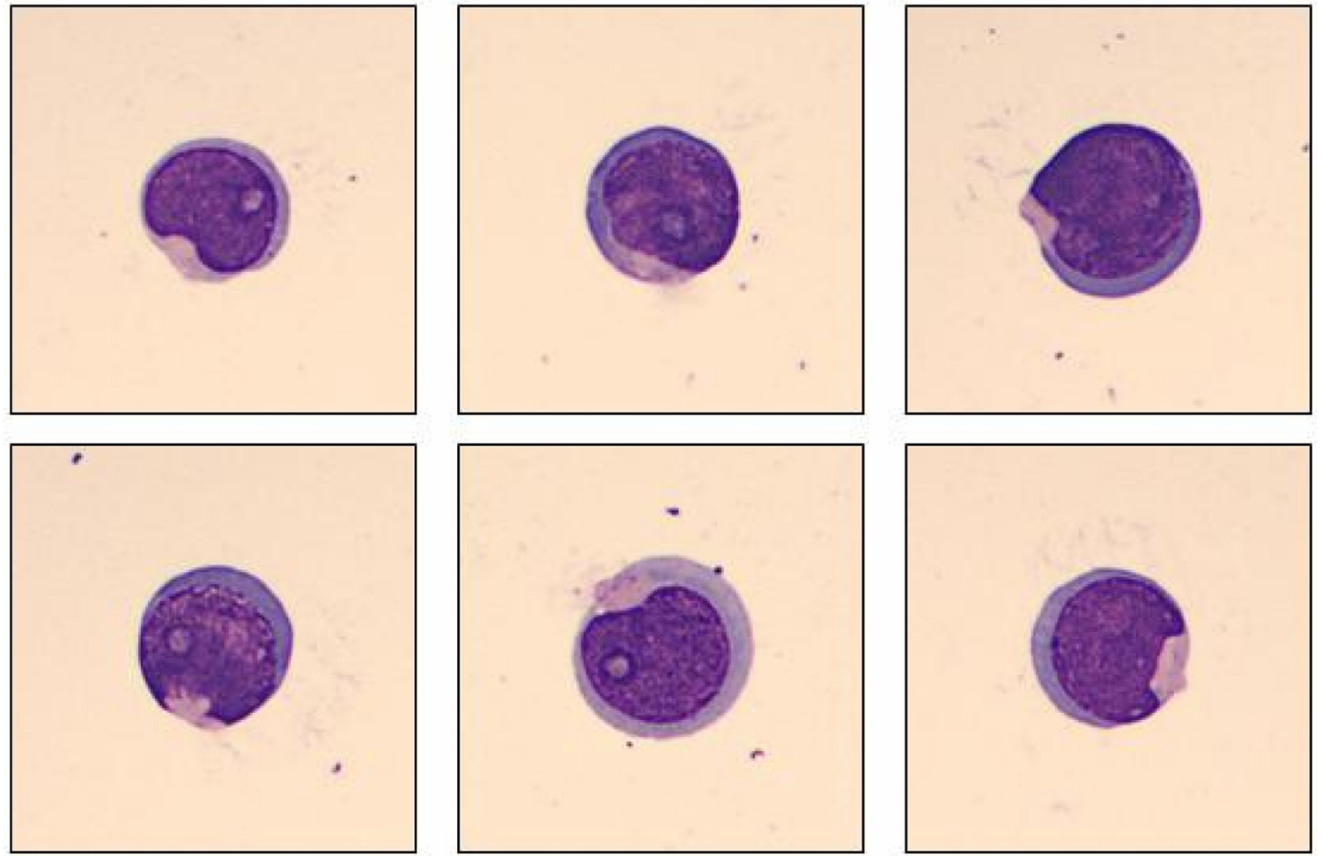

Figure 3 Digital images of blast cells from the pleural effusion, as presented on the digital analyzer.
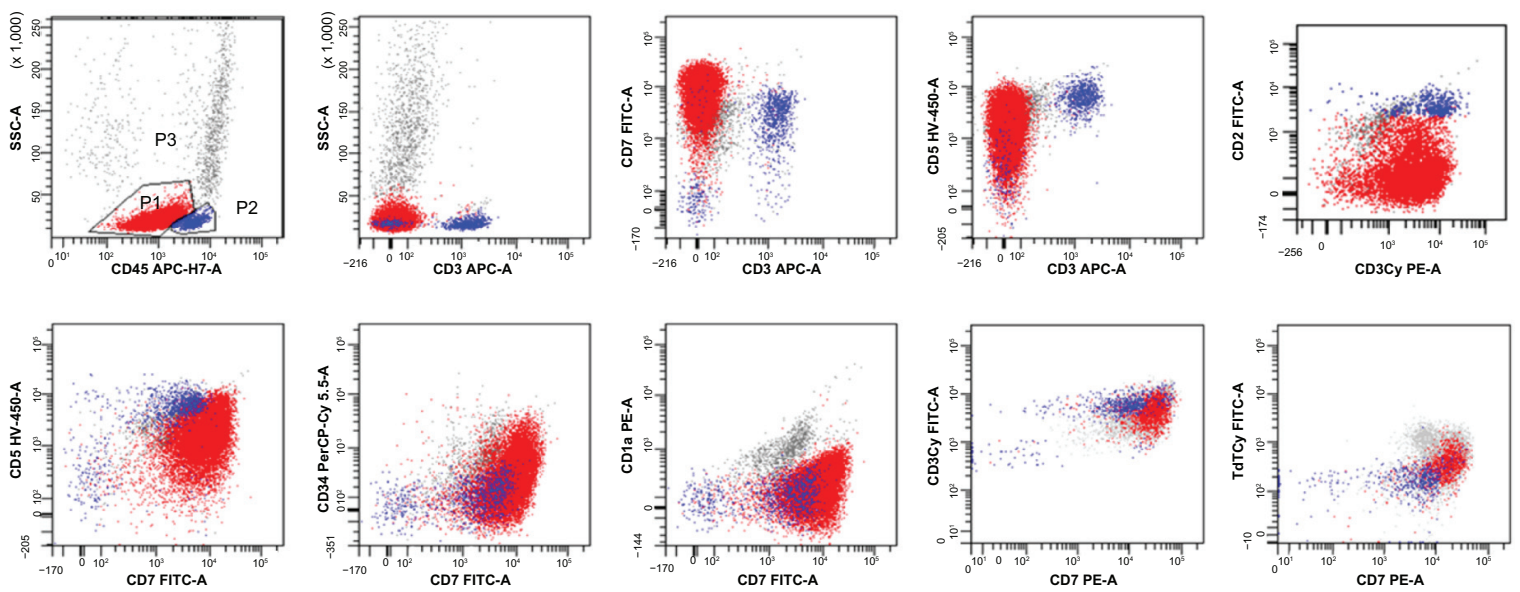

Figure 4 Flow cytometry analysis of the pleural fluid.

Notes: Blasts are shown in red, and lymphocytes are shown in blue. Blasts were CD45+/-, CD3-, CD7++, CD5+, CD2-, CD34+/-, CDIa-, CD3Cy+, and TdTCy+. Abbreviations: SSC, side scatter cells; FITC, fluorescein isothiocyanate; CD3, surface CD3; CD3Cy, cytoplasmic CD3; TdTCy, cytoplasmic TdT.
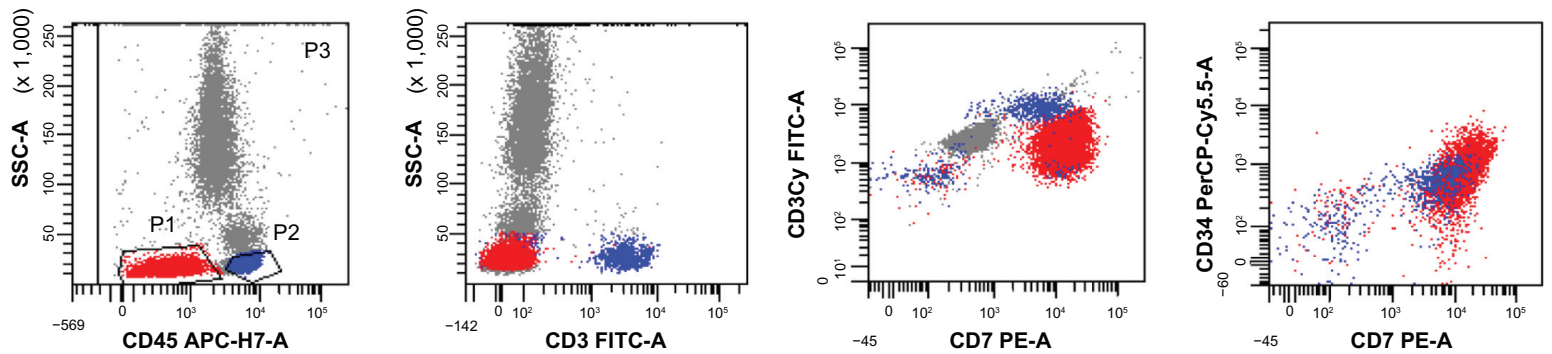

Figure 5 Flow cytometry analysis of peripheral blood.

Notes: Blasts are shown in red, and lymphocytes are show in blue. Blasts were CD45+/-, CD3-, CD7++, CD34+/-, and CD3Cy+.

Abbreviations: SSC, side scatter cells; FITC, fluorescein isothiocyanate; CD3, surface CD3; CD3Cy, cytoplasmic CD3. 


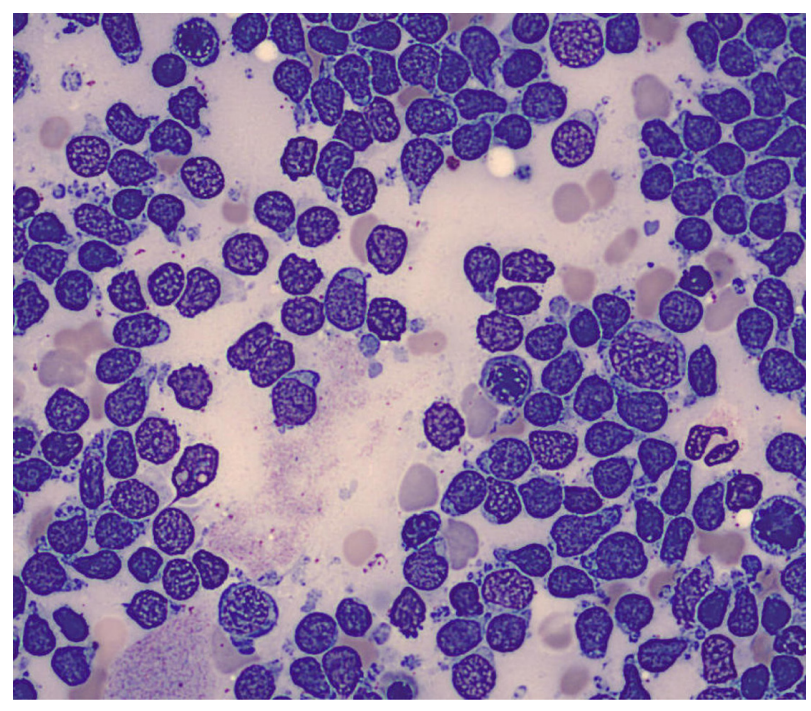

Figure 6 Digital image of bone marrow aspirate.

teria, the malignancy was classified as Pro T-lineage acute lymphoblastic leukemia (T-ALL) (EGIL T1). ${ }^{3}$ Cytogenetic and fluorescence in situ hybridization analyses showed a normal karyotype.

The patient received induction therapy according to the Northern Italy Leukemia Group's protocol. He achieved complete remission on November 2012. Two months later, he received cord blood allogeneic stem cell transplantation and died during aplasia due to septic shock.

\section{Discussion}

ALL is a heterogeneous disease with variable clinical presentation. Clinical picture at diagnosis includes constitutional symptoms (fevers, night sweats, and weight loss), easy bleeding or bruising, dyspnea, dizziness, and infections. Rarely, extremity and joint pain might be the only presenting symptoms. T-ALL with a bulky mediastinal mass can cause stridor and wheezing, pericardial effusions, and superior vena cava syndrome. We reported a case of Pro T-ALL (EGIL T1) presenting with dyspnea and a massive pleural effusion without any hemochrome abnormalities. The analysis of the pleural fluid following thoracentesis revealed blasts and allowed to drive further exams, including immune phenotyping and bone marrow aspirate, leading to a diagnosis of T-ALL.

This case report emphasizes the efficacy of new technology systems and digitalized cell analysis of body fluids to allow for the identification of abnormal lymphoid cells, thus facilitating the ability to reach a proper diagnosis.

\section{Conclusion}

By reporting on this case, we demonstrate the usefulness of the combination of the XE-5000 hematology analyzer and the DM96 as a fast screening system for the morphological assessment of pleural fluid. The DM96 represents a valuable tool that can increase the overall quality of morphological analyses and, as a consequence, increase diagnostic accuracy. We propose the use of the XE-5000 in combination with the DM96 in the diagnostic work-up of body fluid analysis.

\section{Disclosure}

The authors report no conflicts of interest in this work.

\section{References}

1. Paris A, Nhan T, Cornet E, Perol JP, Malet M, Troussard X. Performance evaluation of the body fluid mode on the platform Sysmex XE-5000 series automated hematology analyzer. Int J Lab Hematol. 2010;32(5): 539-547.

2. Riedl JA, Dinkelaar RB, van Gelder W. Automated morphological analysis of cells in body fluids by the digital microscopy system DM96. J Clin Pathol. 2010;63(6):538-543.

3. Szczepański T, van der Velden WH, van Dongen JJ. Classification system for acute and chronic leukaemias. Best Pract Res Clin Haematol. 2003;16(4):561-582.

\section{Publish your work in this journal}

The International Medical Case Reports Journal is an international, peer-reviewed open-access journal publishing original case reports from all medical specialties. Previously unpublished medical posters are also accepted relating to any area of clinical or preclinical science. Submissions should not normally exceed 2,000 words or

\section{Dovepress}

4 published pages including figures, diagrams and references. The manuscript management system is completely online and includes a very quick and fair peer-review system, which is all easy to use. Visit $\mathrm{http}: / / \mathrm{www}$.dovepress.com/testimonials.php to read real quotes from published authors. 